\title{
The Cone Phase of Liquid Crystals: Triangular Lattice of Double-Tilt Cylinders
}

\author{
Yashodhan Hatwalne and N. V. Madhusudana \\ Raman Research Institute, Bangalore - 560 080, INDIA
}

(November 3, 2018)

\begin{abstract}
We predict the existence of a new defect-lattice phase near the nematic-smectic- $C(N C)$ transition. This tilt- analogue of the blue phase is a lattice of double-tilt cylinders. We discuss the structure and stability of the cone phase. We suggest that many 'nematics' exhibiting short range layering and tilt order may in fact be in the molten cone phase, which is a line liquid.
\end{abstract}

61.30.-v, 61.30.Mp, 64.70.Md, 61.30.Jf

In the nematic to smectic- $C(N C)$ transition the continuous translational symmetry of the nematic is spontaneously broken and a layered structure with unit layer normal $\mathbf{N}$ develops [1]. In addition, smectic- $C$ liquid crystals have tilt order which is characterized by the projection $\mathbf{c}$ of the apolar nematic director $\mathbf{n}$ onto the smectic layers. The smectic- $C$ structure belongs to the symmetry group $C_{2 h}$ (a mirror plane, a two-fold rotation axis normal to the mirror plane, and a centre of inversion). Note in particular that this biaxial structure spontaneously breaks the continuous azimuthal symmetry; the average orientation of the $\mathbf{c}$ vector is fixed in space (Fig. 11).

In this letter we address the following question: Can a phase in which the smectic layer normal explores the entire range ( 0 to $2 \pi$ ) of azimuthal angles about the nematic director $\mathbf{n}$ exist? We answer this question in the affirmative, propose a possible defect- lattice structure (Fig. 3) and analyse the mechanism which stabilizes it. Liquid crystals are soft materials; this makes the existence of well-known defect-lattice phases such as the blue phases and the twist grain boundary phases [1] possible. Our discussion has certain similarities with the low chirality stability analysis of the blue phases [1,2]. It is therefore useful to draw an analogy between the stabilizing mechanisms of the blue phases and the cone phase. The cholesteric phase (which has a single twist axis) is unstable to the formation of the blue phases (which are lattices made up of double-twist cylinders) if the coefficient of the saddle-splay term in the molecular director $\mathbf{n}$ in the Frank free energy is positive. The blue phases satisfy the tendency of chiral molecules to sustain twist in all possible directions. The smectic- $C$ phase (which has tilt order in one direction) is unstable to the formation of the cone phase (which is a defect lattice made up of tilt disclinations) if the coefficient of the Gaussian curvature term (which is the saddle-splay- like term in the smectic layer normal $\mathbf{N}$ ) in the free energy is negative. The cone phase is a lattice of double-tilt cylinders in which the smectic layer normal tilts in all possible directions about the nematic director. If the coefficient of the Gaussian curvature term in the free energy is positive in a smectic with tilt order, the cone phase is clearly not stabilized. Instead, we expect 'plumber's nightmare' phases of the type discussed in 3].

Our principal results are as follows: We predict the existence of the cone phase of liquid crystals near the first order $N C$ transition and discuss the mechanism responsible for stabilizing it. The cone phase is a triangular lattice of double-tilt cylinders, each of which is a +1 disclination (in both the c- field as well as the $\mathbf{N}$ - field) in the form of a stack of conical layers. For reasonable values of parameters we estimate that the cone phase composed of double-tilt cylinders of radius $50 \AA$ would be stabilized if the coefficient of the Gaussian curvature term in the free energy $\kappa_{G} \simeq-3 \times 10^{-6}$ dyne. Based on our theoretical analysis and the results of X-ray and neutron diffraction experiments on many compounds exhibiting skew-cybotactic order, we surmise that this so-called 'nematic' phase with short-range layering/tilt order may in fact be the molten cone phase - a line liquid of double-tilt cylinders.

The de Gennes model [4] of the $N C$ transition and the Chen-Lubensky $N A C$ model [5] are motivated by the experimental observation that x-ray scattering shows two rings peaked around $q_{c}=\left(q_{\perp} \cos \phi, q_{\perp} \sin \phi, \pm q_{\|}\right)$in the vicinity of the $N C$ transition. Pretransitional fluctuations clearly explore all the azimuthal angles. Fluctuations drive the second- order mean field $N C$ transition of the Chen-Lubensky model to a first order transition [6,7]. The deGennes model as well as the Chen-Lubensky model predict that all the Frank elastic constants diverge at the $N C$ transition. This has been borne out experimentally [8].

Clearly, any distortion in the nematic director is enormously costly near the $N C$ transition. However, it is possible to have stable configurations where the smectic layer normal exhausts the full range of azimuthal angles relative to a distortion-free nematic director. Such field configurations are topologically nontrivial and necessarily involve disclination lines (Fig. 2). The cone configuration has a positive, delta-function Gaussian curvature along its axis, which screens the disclination charge [9 11]. It is therefore essential to use covariant elasticity to calculate the energetics of the disclination lines. Our formulation naturally incorporates the elasticity theory of membranes with tangent-plane order.

For the stability of the cone phase, it is essential that 
the $N C$ transition be first order (see the discussion following equation (5)). This is the case for almost all compounds exhibiting the $N C$ transition. To describe this transition it is sufficient for our purpose to use a simple phenomenological free energy density

$$
f_{L}=a\left(T_{c}-T\right),
$$

where $a$ is a constant. The free energy density $f_{L}$ accounts for the condensation energy of the smectic layering as well as that for the development of tilt order at the $N C$ transition.

The Frank free energy density

$$
f_{n}=\frac{K_{1}}{2}(\nabla \cdot \mathbf{n})^{2}+\frac{K_{2}}{2}(\mathbf{n} \cdot \nabla \times \mathbf{n})^{2}+\frac{K_{3}}{2}(\mathbf{n} \times \nabla \times \mathbf{n})^{2}
$$

describes the energy cost for distortion in the Frank director (1]. In (2) $K_{1}, K_{2}$ and $K_{3}$ are respectively the splay, twist and bend elastic constants.

The free energy density for distortions in the smectic layering is given by

$$
f_{s}=\frac{B}{2} \gamma^{2}+\frac{\kappa}{2} H^{2}+\kappa_{G} K
$$

where $\gamma$ is the strain due to compression or dilation of the smectic layers, $H$ and $K$ respectively denote the mean curvature and the Gaussian curvature of the smectic layers 伍.

Finally

$$
f_{c}=\frac{K_{A}}{2} D_{i} c^{j} D^{i} c_{j}+\frac{K_{B}}{2}\left(\left(N^{\alpha} \partial_{\alpha}\right) \mathbf{c}\right)^{2},
$$

accounts for the energy cost for distortions in the c- field which do not arise from distortions in the Frank director $\mathbf{n}$ but from distortions in the smectic layers. In (4), $D_{i} c^{j}=\partial_{i} c^{j}+{ }^{(2)} \gamma_{k}^{i} A_{i} c^{k}, A_{i}$ are components of the spin connection defined below, ${ }^{(2)} \gamma_{k}^{i}$ is the completely antisymmetric unit tensor for the two-dimensional smectic layers, $i, j, k=1,2$ refer to the internal coordinates of the smectic layers, and $\alpha=1,2,3$. The spin connection defined via $K={ }^{(2)} \gamma^{i j} \partial_{i} A_{j}$ represents the frustration induced in the c- field on layers with nonzero Gaussian curvature. The term with the coefficient $K_{A}$ in (4) can be schematically written as $\left(K_{A} / 2\right)(S-K) \frac{1}{-\nabla^{2}}(S-K)$, where $S$ is the disclination density and $\nabla^{2}$ is the covariant Laplacian in the two- metric ${ }^{(2)} g_{i j}$ describing the geometry of the smectic layers. Gaussian curvature screens disclination charges 10,11. Stability requires that $B, \kappa, K_{A}, K_{B}>0$. Since Gaussian curvature is a total derivative, the coefficient $\kappa_{G}$ can either be positive or negative. Thus the full free energy for our model is $F=\int\left(f_{L}+f_{n}+f_{s}+f_{c}\right) d V$ [12].

If the coefficient of the Gaussian curvature term in (3) is negative, a double-tilt cylindrical structure of radius $R$ with a +1 disclination in the c- field lowers the energy. However the smectic layers in this conical configuration are bent. We now show that the free energy cost from mean curvature, c- field distortion, and surface tension can be compensated by the free energy gain from Gaussian curvature to stabilize a lattice of disclination cones (Fig. 3).

Let us consider a triangular lattice of cylindrical stacks of cones (i.e., a triangular lattice of straight +1 disclination lines), and fill in the gaps between cylinders with nematic material such that the nematic director is parallel to the disclination cores. Such a configuration minimizes the interfacial energy between the nematic and the smectic. In what follows we ignore the repulsive interaction energy between the disclination lines. In the continuum, a conical stack of smectic layers with its core along the z-axis can be parametrized by a position vector $\mathbf{R}=(r \cos \phi, r \sin \phi, m r+z)$ in the cylindrical polar coordinate system. We choose $m=\tan \theta$, where $\theta$ is the tilt angle of the nematic director with respect to the local layer normal and $D=d / \cos \theta$, where $d$ is the equilibrium smectic layer spacing. This choice ensures that there is no distortion in the nematic director, and that the inter-layer separation along the local layer normal equals $d$. The disclination density is a delta function, $S=2 \pi \delta(r, \phi) / \sqrt{g}$, where $g$ is the determinant of the two-metric. The Gaussian curvature is zero everywhere except on the $z$-axis with a line curvature charge $\Sigma_{+}$: $K=2 \pi \Sigma_{+} \delta(r, \phi) / \sqrt{g}$, with $\Sigma_{+}=1-\frac{1}{\sqrt{1+m^{2}}}$. For cones with radius $R$ the energy per unit length (along the cone axes) of the triangular lattice with reference to the equilibrium smectic- $C$ is

$$
\begin{aligned}
f_{\text {cone }}= & \left(\sqrt{3}-\frac{\pi}{2}\right) a\left(T_{c}-T\right) R^{2} \\
& +\frac{\pi}{2}\left(\kappa \frac{m^{2}}{\sqrt{1+m^{2}}}+K_{A} \frac{m^{2}}{\left(1+m^{2}\right)^{3 / 2}}\right) \ln \frac{R}{a} \\
& +\pi \kappa_{G}\left(1-\frac{1}{\sqrt{1+m^{2}}}\right)+\pi R \sigma,
\end{aligned}
$$

where $a$ is a cutoff length of molecular dimension, and we have included the contribution from the interfacial tension $\sigma$ between smectic- $C$ and nematic. The Frank free energy does not enter into the energetics of the cone phase because the Frank director is free of distortions. Although the Gaussian curvature term in (3) integrates to the boundary, it nevertheless contributes to the energetics because the double-tilt cylinders have a finite radius. The smectic- $C$ - nematic interfaces at the boundaries of the double-tilt cylinders are essential for the stabilization of the cone phase. We also note that the Gaussian curvature term drops out of the energetics for $m=0$. It is therefore clear that double-tilt cylinders cannot be energetically favored near a second-order $N C$ transition in which $m$ grows continuously from zero in the smectic$C$ phase. In fact a relatively strong first order transition 
with sufficiently small (large in magnitude and negative in sign) value of $\kappa_{G}$ would favor the formation of stable double-tilt cylinders.

We assume the following reasonable values for the parameters entering (5): $\kappa \simeq 10^{-6}$ dyne, $K_{A} \simeq 3 \times 10^{-7}$ dyne, $\sigma \simeq 10^{-2}$ dyne/cm 13. At the first order $N C$ transition point there is no free energy difference between the two phases. With $m=1 / 2$ [14, double-tilt cylinders of radius $R \leq 50 \AA$ are energetically favored over a distortion free smectic- $C$ provided $\kappa_{G} \leq-3 \times 10^{-6}$ dyne. We are not aware of any measurements of $\kappa_{G}$ in thermotropic liquid crystals. However, some lyotropic liquid crystals have $\kappa_{G}$ values of this sign and magnitude [15]. It is clear that the energy of a double-tilt cylinder is lowered for smaller values of $R$. It is interesting to contrast this with the case of blue phases of cubic symmetry where the pitch of the helix provides a natural length scale which determines the radius of double-twist cylinders. There is no such natural length scale determining the radius of double-tilt cylinders of the cone phase 116]. We therefore expect the radius of the double-tilt cylinders to be of the order of a few times the cutoff length, which is the smallest possible radius consistent with the conical structure.

Purely from a molecular point of view the tilt of the molecular director in the smectic- $C$ owes its origin to the fact that a mutual displacement of the molecules along their long axes lowers the interaction energy 17]. If all the neighbors of a given molecule are displaced relative to it by an equal amount in the same direction, the interaction energy can be lowered further. This is precisely the molecular configuration near the apex of the cones forming double-tilt cylinders. This tendency of the molecules may contribute significantly towards rendering $\kappa_{G}$ negative.

Narrow double-tilt cylinders arrange themselves in a triangular lattice to form the cone phase. With a decrease in temperature from the transition point, the energy cost for the nematic which fills the gap between the cylinders would become prohibitively large. Consequently there would be a transition from the cone phase to the smectic$C$ phase. We expect the temperature range of the cone phase to be narrow (of the order $1^{0} \mathrm{C}$ ) as in the case of the blue phases. To our knowledge such a phase has not been reported in the literature. However there are several examples of compounds which exhibit a skew-cybotactic short-range order over a wide (about $50^{\circ} \mathrm{C}$ ) temperature range [18,19]. X-ray and neutron scattering experiments show that these systems exhibit several orders of scattering maxima corresponding to molecular layering along the nematic director in addition to the scattering due to tilt-order in the layers. This implies that the molecular positions are correlated along the director. This scattering pattern corresponding to fiber-like structures can be interpreted as arising from the narrow double-tilt cylinders proposed above. Since the medium is in the ne- matic phase, there is no long-range positional order of the double-tilt cylinders. Indeed, these 'nematics' may in fact be in a molten line liquid phase which has been discussed in the context of directed polymers in nematics, and flux lattices 20]. As is the case for flux tubes in superconductors, double-tilt disclination lines have a purely repulsive logarithmic interaction potential. The melting of the cone phase should therefore be analogous to the melting of the Abrikosov flux lattice, where the 'braiding entropy' from the entanglement of the flux lines wins over the potential energy cost at the melting point. Detailed analysis of the melting of the cone phase into the line liquid phase is outside the scope of this letter. We note that the foregoing analysis is also applicable to structures such as the smectic- $I$ which has hexatic order with molecular tilt oriented along bonds connecting nearest neighbors [1].

Further experiments to confirm the work presented in this letter are clearly of great interest. We have taken up some experiments to test these ideas in our laboratory.

We thank V. A. Raghunathan, Madan Rao and Joseph Samuel for useful discussions.

[1] See P. G. deGennes and J. Prost, The Physics of Liquid Crystals ( Clarendon Press, Oxford, 1993) and references therein.

[2] S. Meiboom, J. P. Sethna, P. W. Anderson, and W. F. Brinkman, Phys. Rev. Lett. 46, 1216(1981).

[3] A. M. Levelut and Y. Fang, J. de Physique 51, C7$229(1990)$.

[4] P. G. deGennes, Mol. Cryst. Liquid Cryst. 21, 49(1973).

[5] J.-H. Chen and T. C. Lubensky, Phys. Rev. A 14, 1202 (1976)

[6] S. A. Brazovskii, Zh. esp. teor. Fiz.68 175, Sov. Phys. JETP 41, 85(1975).

[7] J. Swift, Phys. Rev. A14, 2274(1976).

[8] S. Witanachi, J. Huang, and J. T. Ho, Phys. Rev. Lett. 50, 594(1983).

[9] D. R. Nelson and L. Peliti, J. Phys. (Paris) 48, 1085(1987).

[10] M. W. Deem and D. R. Nelson, Phys. Rev. E 53, 2551(1996).

[11] J. -M. Park and T. C. Lubensky, Phys. Rev. E 53, 2648(1996).

[12] The volume element $d V$ contains hidden factors of the three dimensional metric. Smectic structure naturally slices 3-space into 2-surfaces (the smectic layers). The 3metric can be expressed in terms of the 2-metric on the 2-surfaces, a normal shift function and a tangent shift vector. This is analogous to the slicing of curved spacetime in canonical general relativity. See, for example, C. W. Misner, K. S. Thorne, and J. A. Wheeler, Gravitation (Freeman, 1970).

[13] The smectic- $A$ - nematic interfacial tension is of this or- 
der. See S. Faetti and V. Palleschi, J. Chem. Phys. 81, $6254(1984)$. We expect the smectic- $C$ - nematic interfacial tension to be of the same order of magnitude.

[14] A. de Vries, Mol. Cryst. Liq. Cryst. 10, 31(1970).

[15] See A. G. Petrov, The Lyotropic State of Matter (Gordon and Breach, Amsteldijk, 1999) and references therein.

[16] We note that this is strictly correct only for the model discussed in this letter, where we have ignored higher order terms in the elastic free energy.

[17] A. S. Govind and N. V. Madhusudana, Europhys. Lett. 55, 505(2001).

[18] K. Usha Deniz, G. Pepy, P. Keller, B. Farnoux and G. Paratte, Mol. Cryst. Liq. Cryst. 127, 81(1985)

[19] N. V. Madhusudana, K. P. L. Moodithaya and K. A. Suresh, Mol. Cryst. Liq. Cryst. 99 239(1983).

[20] D. R. Nelson in Observation, Prediction and Simulation of Phase Transitions in Complex Fluids, Ed. M. Baus, L. F. Rull and J. -P. Ryckaert ( Kluwer, Dordrecht, 1995).

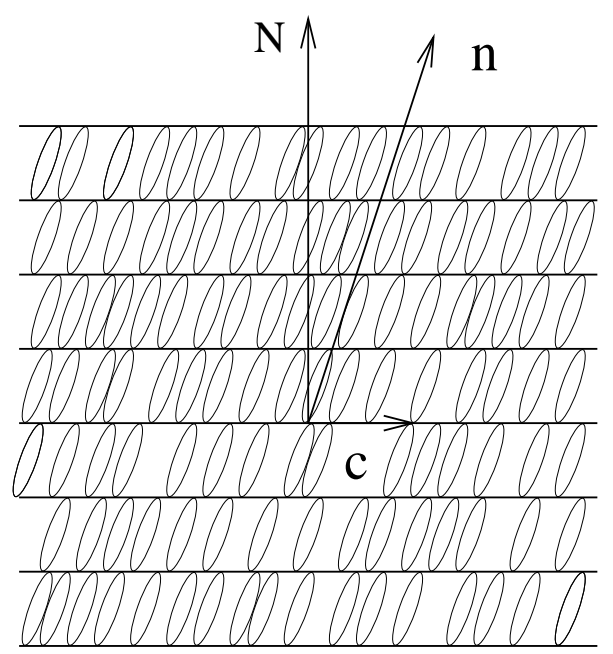

FIG. 1. Schematic of the equilibrium smectic- $C$ structure.

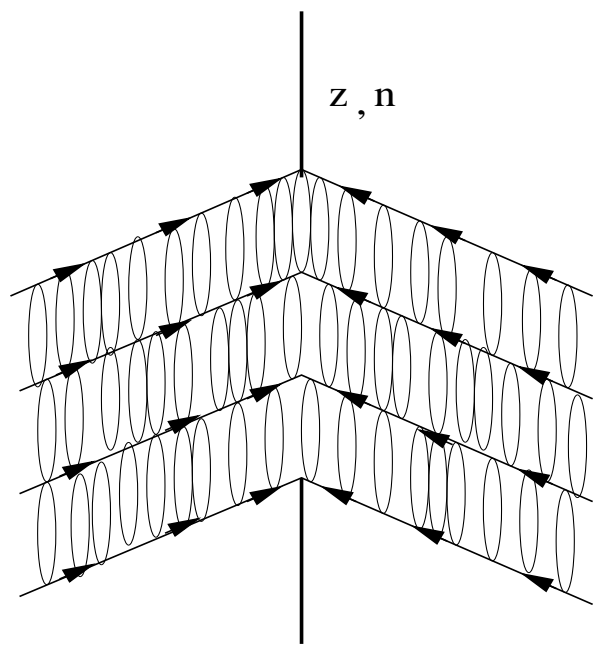

FIG. 2. Schematic of a double-tilt cylinder. The nematic director $\mathbf{n}$ is parallel to the cone axis, which is a +1 disclination line. The c- vector (shown by the arrows in the figure) lies in the smectic layers and points towards the cone axis. Note that the n- field has no distortion, whereas the smectic layers bend. There is no change in the smectic layer spacing along the local layer normal.

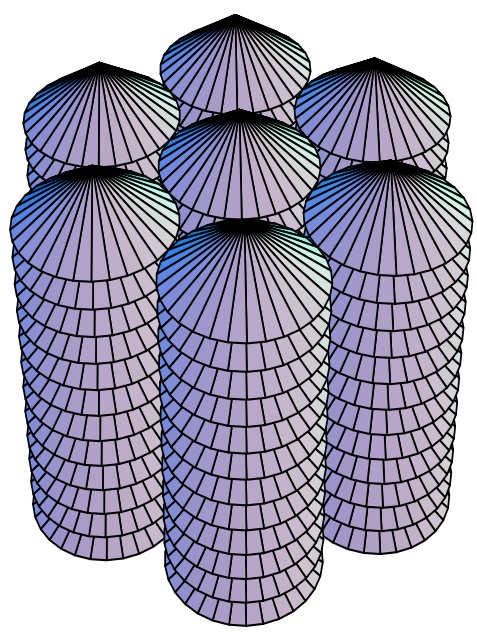

FIG. 3. Schematic of the cone phase - a triangular lattice of double-tilt cylinders. Each cylindrical stack of cones is a double-tilt cylinder depicted in (Fig. 2). 